\title{
Multiple Sclerosis and Regulatory T Cells
}

\author{
Cristina Maria Costantino, Jonathon Hutton, Clare Baecher-Allan, and David A. Hafler \\ Division of Molecular Immunology, Center for Neurologic Diseases, Brigham and Women's Hospital, \\ and Harvard Medical School, Boston, Massachusetts, 02115
}

\begin{abstract}
Multiple sclerosis (MS) is a complex genetic disease characterized by chronic inflammation of the central nervous system (CNS). The pathology of MS is largely attributed to autoreactive effector T cells that penetrate the blood-brain barrier and become activated within the CNS. As autoreactive T cells are present in the blood of both patients with MS and healthy individuals, other regulatory mechanisms exist to prevent autoreactive $\mathrm{T}$ cells from causing immune disorders. Active suppression by regulatory $\mathrm{T}$ (Treg) cells plays a key role in the control of self-antigen-reactive $\mathrm{T}$ cells and the induction of peripheral tolerance in vivo. In particular, the importance of antigen-specific Treg cells in conferring genetic resistance to organ specific autoimmunity and in limiting autoimmune tissue damage has been documented in many disease models including MS. We have found that the frequency of Tregs in MS patients is unchanged from controls, but their function measured in vitro may be diminished, correlating with impaired inhibitory activity in vivo. This review discusses the immunopathology of MS with particular focus given to regulatory $\mathrm{T}$ cells and their potential for the development of new therapies to treat this disease.
\end{abstract}

\section{Introduction}

Multiple sclerosis (MS) is a complex genetic disease associated with chronic inflammation of the CNS, demyelination, axonal loss, and brain atrophy. MS has a strong genetic basis, which has been confirmed in several large population-based MS twin studies; the sibling-topopulation risk $\left(\lambda_{S}\right)$ of developing MS is 40 (type 1 diabetes, a complex genetic disease with greater environmental risk factors, has a $\lambda_{S}$ of only 15$)[1,2,3]$. These genetic risk factors manifest in autoimmune inflammation,

Evidence of the inflammatory nature of MS dates back to 1948, when Elvin Kabat observed that MS patients have abnormally high levels of oligoclonal immunoglobulin in the cerebrospinal fluid (CSF) [4]. Self-antigen directed inflammation is thought to precede development of MS; this relationship has been demonstrated in experimental autoimmune encephalomyelitis (EAE), an animal model of MS [5]. In this animal model, the minimal requirement for inducing CNS inflammation is activation of myelin reactive T cells in the peripheral immune system [6]. Activated, autoreactive T lymphocytes also regulate MS pathophysiology. Blocking lymphocyte egress from the vascular compartment into the CNS with agents such as Natalizumab ( $\alpha \mathrm{VLA}-4 \mathrm{mAb})$ reduces many symptoms of MS and slows the progression of the disease [7]. In this chapter, we discuss the pathophysiology of MS, with a focus on the role of $\mathrm{CD}^{+} \mathrm{T}$ cells in establishing and regulating immune inflammation. 


\section{Immunopathophysiology of MS}

Autoreactive T cells recognizing myelin antigens are present in the circulation of both MS patients and healthy individuals. Activation of these T cells is critical for autoimmune disease induction. In the EAE model, injection of healthy mice with myelin antigen and adjuvant is sufficient to induce disease in genetically susceptible strains. In humans, $\mathrm{T}$ cell clones specific for myelin antigen can be isolated from peripheral blood.

Several years ago, our lab and others cloned $\mathrm{T}$ cells from the blood of patients with MS to query the antigen specificity of these cells. Among the T cell clones we isolated, several exhibited exquisite specificity for MBP p85-99, an immunodominant epitope first detected in EAE mice [8-10]. The T cell receptor (TCR) for one such clone, Ob.1A12, has been successfully crystallized and was found to bind in an unconventional fashion to p85-99 in complex with autologous HLA-DR. The primarily contact points for the Ob.1A12 TCR were common determinants in the backbone of the MHC class II binding pocket and not side chains extending from the peptide antigen. Therefore, the binding affinity of the Ob.1A12 TCR for the HLA-DR:peptide complex had less reliance on the identity of the peptide than a non-selfreactive T cell clone with a conventional TCR binding pattern [11]. Nonconventional TCR binding allows for peptide antigen flexibility, but increased TCR affinity for the MHC class II binding pocket is required in order to maintain specificity.

It follows, therefore, that autoreactive $\mathrm{T}$ cell clones would demonstrate a significant degree of functional degeneracy in antigen recognition, and this is in fact the case. Cross-reactivity against other myelin antigens, such as myelin oligodendrocyte protein (MOG), has been observed in MBP-reactive T cell clones and alteration of the peptide ligand has been shown to improve T cell clone reactivity [12]. Furthermore, recent studies characterizing TCR degeneracy have determined that the frequency of T cells with degenerate TCR is higher in patients with MS than in healthy controls, and that a subset of these cells recognize myelin antigen [13]. These findings support the hypothesis cross-reactive $\mathrm{T}$ cells in the brain are activated by non-self antigen during the course of an infection.

Such "molecular mimicry" by viral or bacterial proteins could initiate autoimmune pathogenesis by myelin-reactive T cells [14,15], Karl-Heinz Wiesmüller and colleagues have used a combinatorial chemistry approach to provide evidence for this hypothesis and have succeeded in identifying a number of viral epitopes that trigger autoreactive T-cell clones, including a peptide from human cytomegalovirus [16]. Other infectious agents, including Epstein-barr virus (EBV), Chlamydia pneumoniae, hepatitis B virus, and Haemophilus influenzae, have also been reported to express cross-reactive epitopes [17,18,19,20]. Molecular mimicry during the course of an infection could instigate development of MS in genetically susceptible individuals. Yet, to date there is little direct support providing a causal link between infection and MS. The best epidemiological candidate to date is EBV, which has a slightly higher incidence rate in MS patients (100\%) than in healthy controls (90\%) [21]. A recent study by Ascherio and coworkers, however, suggested that incidence of EBV infection, as measured by EBV seropositivity, was linked to HLA haplotype [22]. Common genetic risk factors, rather than an etiological role for EBV infection in MS, could explain the shared frequency of incidence in these two diseases.

Although the antigen(s) that initiate T cell activation in MS remain unknown, studies using EAE mice have provided some insight into the cascade of myelin-directed responses that characterize T cell pathology in the CNS. Indeed, the immunodominance of a disease-initiating peptide epitope may be restricted to the inductive phase of the immune reaction. Sercarz and colleagues provided evidence for this hypothesis using genetically susceptible SJL x B10.PL) F1 mice, which develop EAE in response to injection with the single myelin protein epitope, 
MBP(Ac1-11)[23], These mice generated a $\mathrm{CD}^{+} \mathrm{T}$ cell-directed response against the injected peptide antigen, but during the course of the disease $\mathrm{T}$ cells in these mice also become activated against other epitopes of MBP. When isolated and adoptively transferred into naïve mice, $\mathrm{T}$ cells specific for cryptic MBP epitopes were sufficient to initiate disease. This phenomena, known as "epitope spreading", is also thought to occur in MS, as most patients exhibit T cell reactivity to a number of myelin antigens [8]. In a transgenic mouse expressing human HLADR (DRB1*1501) and the Ob.1A12 TCR, spontaneous development of EAE was accompanied by epitope spreading to a number of antigens implicated in MS, including $\alpha$-crystalline and proteolipid protein (PLP) [24]. Determination of the disease-initiating immunodominant epitope in human disease may prove near impossible, however, as it is likely that by the time a patient develops clinical MS epitope spreading has already occurred,

\section{CNS-Specific regulation of inflammation}

The CNS is an immune-privileged site with unique anatomy and cell types of that limit inflammation and autoimmunity. Recent advances in neurobiology and immunology, aided by technical advances in gene expression analysis and in vivo imaging, have begun to provide greater insight into the molecular events occurring within and around MS plaques.

Understanding these mechanisms is critical in determining the role that resident cells in the CNS play in regulating inflammation.

\section{Cellular events occurring within and around MS plaques}

Gross examination of MS brain tissue reveals multiple sharply demarcated plaques in the CNS white matter with a predilection to the optic nerves and white matter tracts of the periventricular regions, brain stem, and spinal cord. As was recognized early on and so elegantly investigated in more recent studies, substantial axonal injuries with axonal transactions are abundant throughout active MS lesions [25].

There are four pathologic categories of MS related to potentially different pathophysiologic disease mechanisms, though this has yet to be demonstrated at a molecular level [26]. These categories include acute, relapsing/remitting, primary progressive and secondary progressive forms of the disease. Within a single diseased individual, the pattern of pathology tends to be the same, even if the patient exhibits multiple lesions.

The inflammatory cell profile of active lesions is characterized by perivascular infiltration of monocytes, oligoclonal T cells [27] -both $\mathrm{CD}^{+} / \mathrm{CD} 8 \alpha / \beta[28,29]$ and $\gamma \delta[30]$-and less frequently, B cells and plasma cells [31]. Lymphocytes can also be found in normal appearing white matter beyond the margin of active demyelination [32]. Macrophages are most prominent in the center of the plaques and are seen to contain myelin debris. In chronic active lesions, the inflammatory cell infiltrate is less prominent and may be largely restricted to the rim of the plaque, suggesting the presence of ongoing inflammatory activity along the lesion edge.

\section{Regulation of CNS inflammation by TIM3 expression on microglia}

Increasingly, focus is shifting to the roles of glial cells in regulation of CNS inflammation $[33,34]$. Glial cells may respond in predetermined manners to inflammatory cues, with the glial responses having indirect but profound consequences on neurons and infiltrating lymphocytes. Our lab speculated that TIM-3, a molecule originally identified on Th1 cells that selectively limits their function, might be expressed on microglia and influence CNS inflammation.

We first examined TIM-3 expression in the CNS of autopsy tissue from subjects with no known inflammatory disease. We observed robust TIM-3 staining in white matter but not grey matter parenchyma on what appeared histologically to be microglia. We stained these cells with 
CD11b to confirm that TIM-3 was indeed expressed only on microglia in the CNS white matter. Although TIM-3 was differentially expressed, HLA-DR staining was comparable on both white matter and grey matter microglia, suggesting that these two populations are comparable as antigen-presenting cells (APCs). We confirmed the specific and selective expression of TIM-3 on white matter microglia using both laser capture microdissection (LCM) and quantitative RT-PCR analysis. Little or no TIM-3 mRNA was detected in microglia obtained from grey matter tissue whereas we observed significant TIM-3 mRNA in microglia obtained from white matter tissue [34]. Selective expression of TIM-3 in the white matter suggests a role for TIM-3 in modulating the function of lymphocyte infiltrate.

Our research has indicated that TIM-3 on human monocytes triggers TNF- $\square$ production (unpublished observation). Given the known deleterious effects of TNF- $\square$, we speculated that TIM-3-mediated TNF- $\alpha$ production might contribute to CNS tissue pathology. We examined TIM-3 expression on infiltrating monocytes/microglia isolated from the white matter CNS tissue of patients with MS and from CNS glioblastoma multiforme (GBM) tumors. While lymphocytes and monocytes/microglia are present in both types of inflamed tissues, the cytokine profiles differ considerably: the Th 1 cytokines, IFN- $\gamma$ and TNF- $\alpha$, are associated with MS but not GBM tissues. We found that monocytes/microglia captured from the active border regions of MS lesions expressed higher levels of TIM-3 than did those captured from the quiescent center of MS lesions, adjacent normal appearing white matter, or those obtained from non-inflamed white matter tissue. In contrast, TIM-3 expression was significantly lower in monocytes/microglia obtained from GBM tissues relative to those obtained from control tissue or MS tissue.

While there is a small degree of grey matter involvement, MS is predominantly a disease of CNS white matter. It has been widely assumed that MS is a white matter disease because antigen specific cells recognize their antigens predominantly within CNS white matter. While we believe this is fundamentally true, these data raise an interesting hypothesis: myelin-reactive Th1 cells enter white matter regions of the CNS, recognize myelin antigens and secrete IFN$\gamma$, inducing high levels of galectin-9 on astrocytes. Galectin-9 is the only known ligand for TIM-3. During the early remitting-relapsing phase of disease, galectin- 9 expression may effectively terminate Th1 cells responsible for driving inflammation. At later time points, when microglia and DCs become activated and more prevalent at inflammatory sites, expression of galectin- 9 on reactive astrocytes may engage TIM-3 on the surface of microglia and/or infiltrating monocytes/DCs. Ligation of TIM-3 in the white matter then induces TNF- $\alpha$ secretion and promotes secondary progressive disease. In addition, secretion of IL-1 by activated APCs within the CNS could help maintain galectin-9 expression on reactive astrocytes and perpetuate this inflammatory loop.

\section{Toll-like receptor expression on microglia}

Lipopolysaccharide (LPS), a molecular component of Gram-negative bacteria, has been shown to induce neuronal and axonal loss both in vitro and in vivo due to activation of microglia through Toll-like receptor 4 (TLR-4) [35]. Peripheral administration of LPS to female rats resulted in a $240 \%$ increase in the density of activated microglia in the dentate gyrus of the hippocampus, which correlated with a 35\% decrease in hippocampal neurogenesis [36]. Coculture of neural progenitor cells with LPS-stimulated but was not observed not resting microglia inhibited neurogenesis in vitro by approximately $50 \%$, an effect that was mediated by microglial secretion of IL-6. The effect of peripheral LPS administration on neurogenesis was completely blocked by systemic administration of the non-steroidal anti-inflammatory drug indomethacin. Similar results have been obtained by Lindvall and colleagues, who documented an inverse correlation between the number of activated microglia and new neurons in the hippocampus after CNS delivery of LPS [37]. Again, suppression of microglia activation, 
this time with systemic administration of minocycline, reduced the number of activated microglia and increased the number of new neurons in the hippocampus.

In MS lesions, IL-6 is up-regulated in active lesions relative to inactive lesions [38,39] and could contribute to axonal destruction. Indeed, IL-6-deficient mice are completely resistant to EAE [29]. While both microglia and astrocytes become activated in response to CNS inflammation, they may respond differently to inflammatory stimuli. For example, microglia but not astrocytes are responsible for production of the Th1-promoting cytokine IL-12 [33]. Aloisi et al. [40] have demonstrated that astrocytes inhibit IL-12 secretion by in vitro activated microglia, while others have demonstrated using primary astrocyte/microglia co-cultures that astrocyte-derived IL-10 inhibits LPS-induced secretion of the proinflammatory molecules nitric oxide (NO), IL-6, tumor necrosis factor-a (TNF- $\alpha$ ), and IL-1b [41]. Orian and colleagues [42] have recently demonstrated that depending on the mouse strain used (NOD/Lt or C57B1/ 6), injection with encephalitogenic myelin oligodendrocyte glycoprotein (MOG) peptide 3555 induces disease that resembles relapsing/remitting or primary progressive forms of MS. Subsequently, they have found that in the relapsing/remitting form of disease induced in NOD/ Lt mice, there is evidence of both astrocyte and microglial activation prior to the first clinical sign of disease [43]. In contrast, microglia but not astrocytes appear activated in the primary progressive form of disease induced in C57B1/6 mice. While preliminary, the data suggest that differences in microglial and astrocytic responses to inflammation could influence the pathogenesis of MS.

Astrocytes may also prove to indirectly suppress CNS inflammation by induction of regulatory populations of $\mathrm{T}$ cells. A recent report demonstrated that primary rat astrocytes (or astrocytoma cell lines) cultured for $24 \mathrm{~h}$ with primary $\mathrm{T}$ cells inhibited T-cell secretion of interferon (IFN)$\gamma$ in a cell contact-dependent manner [44]. T cells cultured with astrocytes in turn acquired a regulatory phenotype in that they could inhibit Con A-induced T-cell proliferation in vitro and ameliorate the severity of EAE induced with rat spinal cord homogenate in vivo. Using ex vivo human malignant glioma tumor specimens, we have recently demonstrated that astrocytederived tumor cells are notable for their secretion of the immunosuppressive cytokines IL-10 and TGF- $\beta$ and that $\mathrm{CD} 4{ }^{+} \mathrm{CD} 25^{+}$effector cells that infiltrate the tumors are notable for their secretion of IL-10 in the near absence of IFN- $\gamma$. Due to their propensity to secrete IL-10, it is tempting to speculate that astrocytes may naturally promote generation of IL-10-secreting type $1 \mathrm{~T}$-regulatory cells that could help suppress CNS inflammation [45]. It remains to be seen whether nontransformed reactive astrocytes can similarly induce regulatory populations of lymphocytes and in which CNS inflammatory diseases this may arise.

\section{Natural regulatory $\mathrm{T}$ cells in MS}

Clonal deletion of self-reactive $\mathrm{T}$ cells in the thymus and induction of T-cell anergy alone do not explain the maintenance of immunologic self-tolerance, as potentially pathogenic autoreactive $\mathrm{T}$ cells are present in the periphery of healthy individuals $[8,46]$. Thus, other regulatory mechanisms exist to prevent autoreactive $\mathrm{T}$ cells from causing immune disorders. Active suppression by regulatory $\mathrm{T}$ cells plays a key role in the control of self-antigen-reactive $\mathrm{T}$ cells and the induction of peripheral tolerance in vivo [47,48]. Seminal experiments performed by Sakaguchi et al. [47] have shown that depletion of $\mathrm{CD} 4^{+} \mathrm{CD} 25^{+}$suppressor cells results in the onset of systemic autoimmune diseases in mice. Furthermore, co-transfer of these cells with $\mathrm{CD} 4{ }^{+} \mathrm{CD} 25^{-}$cells prevents the development of experimentally induced autoimmune diseases such as colitis, gastritis, insulin-dependent autoimmune diabetes, and thyroiditis [50]. 


\section{Cell surface characterization of human Tregs}

Our group and others described a population of $\mathrm{CD} 4{ }^{+} \mathrm{CD} 25^{\mathrm{hi}}$ regulatory $\mathrm{T}$ cells in human peripheral blood and thymus [51,52]. Human $\mathrm{CD} 4{ }^{+} \mathrm{CD} 25^{\mathrm{hi}} \mathrm{FoxP}^{+}$natural regulatory $\mathrm{T}$ cells (Treg), similar to the mouse $\mathrm{CD} 4{ }^{+} \mathrm{CD} 25^{+}$suppressor cells, are anergic to in vitro antigenic stimulation and strongly suppress the proliferation of responder $\mathrm{T}$ cells upon coculture. Although both murine and human $\mathrm{CD} 4{ }^{+} \mathrm{CD} 25^{\text {high }} \mathrm{FoxP}^{+}$Treg similarly suppress the activation of $\mathrm{CD} 4{ }^{+} \mathrm{CD} 25^{-}$responder $\mathrm{T}$ cells (Tresp) in a cell contact dependent manner and express the Treg-specific lineage specification factor FoxP3, the human Treg population is by far more heterogeneous than that of the mouse, as gauged by both cell surface phenotype and functional capability. Human blood, isolated from an out bred population in a pathogenic environment, contains up to $30 \% \mathrm{CD} 4{ }^{+} \mathrm{CD} 25^{+}$cells; only the $2-4 \%$ of the cells with the highest CD25 expression can be considered regulatory [51]. Problematically, there is no consensus as to where the boundary lies between CD25 high and CD25 intermediate expression, which has hindered both experimental reproducibility and clinical analysis of patients' blood. This is particularly concerning in patients where inflammatory conditions can lead to an increase in CD25 expression by activated T cells. The majority of human Treg can, however, be differentiated from recently activated effector cells by CD62L (L-selectin) expression because $\mathrm{CD} 4{ }^{+} \mathrm{CD} 25^{\text {high }}$ Treg are predominantly $\mathrm{CD} 62 \mathrm{~L}^{+}(>95 \%)$. Additionally, human Treg isolated from adult peripheral blood or tonsils are predominantly $\mathrm{CD}_{4} 5 \mathrm{RO}^{+}, \mathrm{CD} 45 \mathrm{RA}^{-}$, and $\mathrm{CD} 45 \mathrm{RB}^{\text {low }}$ [52]. For the most part, $\mathrm{CD} 4^{+} \mathrm{CD} 25^{\text {high }}$ Treg express a highly differentiated central memory phenotype and share many of the cell surface markers expressed on chronically activated $\mathrm{CD} 4^{+} \mathrm{T}$ cells.

The $\mathrm{CD} 4{ }^{+} \mathrm{CD} 25^{\text {high }}$ Treg population is also enriched for many immunomodulatory surface markers, including MHC class II, CD95 (Fas), glucocorticoid-induced tumor necrosis factor receptor family-related protein (GITR), and cytotoxic T lymphocyte-associated protein (CTLA-4) that are not Treg specific. The most specific marker for Treg to date is the nuclear transcription factor FoxP3, the expression of which correlates with suppressive ability. In humans, FoxP3 is expressed strongly in $\mathrm{CD} 4{ }^{+} \mathrm{CD} 25^{\text {high }} \mathrm{T}$ cells and at low levels in activated $\mathrm{CD}^{+} \mathrm{T}$ cells [53], although FoxP3 expression has also been reported in a small fraction of the $\mathrm{CD} 25^{-}, \mathrm{CD} 25^{\text {intermediate }}$, and CD8 ${ }^{+} \mathrm{T}$ cell subsets. Indeed, data from FoxP3-GFP reporter mice has shown that $\mathrm{CD}^{2} 5^{-} \mathrm{FoxP}^{-} \mathrm{GFP}^{+}$cells exhibit the same suppressive capacity as $\mathrm{CD}^{2} 5^{+} \mathrm{FoxP} 3-\mathrm{GFP}^{+}$cells in vitro [54]. This evidence, paired with the absence of a unique Treg cell surface marker, suggests the possibility that the $\mathrm{CD} 4{ }^{+} \mathrm{CD} 25^{\text {high }}$ subset is merely enriched for human Treg and does not contain the entire Treg population.

Discovery of an alternative marker to CD25 would greatly enhance the identification and purification of human Treg. The best-accepted alternative is lack of cell curface CD127 (IL-7 receptor). Foxp3 expression and suppressive capacity have been detected in $\mathrm{CD} 4^{+} \mathrm{T}$ cells expressing low levels of CD127 [55,56]. Certainly, low CD127 expression may be useful in isolating FoxP3 ${ }^{+}$cells from the $\mathrm{CD} 25^{+} \mathrm{DN} 2$ and DN3 populations of the thymus. In the periphery, however, the possibility remains that CD127 expression does not discriminate between adaptive Treg cells, such as the $\operatorname{Tr} 1$ and $\operatorname{Th} 3$ subsets, activated T cells, and thymicallyderived Tregs.

An alternative marker to CD25 would also be useful for distinguishing tissue-resident Tregs from activated Tresp in inflammatory environments. Ruprect and coworkers have suggested that Tregs from the synovial fluid of patients with juvenile arthritis are $\mathrm{CD} 27^{+}$as well as $\mathrm{CD} 4^{+} \mathrm{CD} 25^{+}$[57]. This population of Tregs expressed elevated levels of FoxP3 and suppressed Tresp proliferation more efficiently than $\mathrm{CD} 4^{+} \mathrm{CD} 25^{+} \mathrm{CD} 27^{-}$synovial fluid Tregs. This study did not restrict the Treg pool to the CD25 ${ }^{\text {high }}$ population, however, so it is difficult to know whether suppressive $\mathrm{CD} 27^{+}$cells are members of this enriched Treg population. 


\section{MHC Class II expression delineates a unique subset of human Tregs}

One of the most striking heterogeneities of the $\mathrm{CD} 4^{+} \mathrm{CD} 25^{\text {high }}$ regulatory population is the expression of HLA-DR in approximately one-third of human Tregs [51]. Although presentation of antigen via HLA-DR by CD4 ${ }^{+} \mathrm{T}$ cells is associated with a profound degree of anergy, contact-dependent suppression by Tregs is not MHC restricted. Therefore T cell presentation of antigen is not a mechanism of regulation in this cell population; rather, HLA-DR expression identifies a functionally distinct population of what appear to be terminally differentiated human Tregs. FoxP3 expression is significantly higher in the HLA-DR ${ }^{+}$Tregs [58]. These cells exhibit earlier kinetics of Tresp suppression than the HLA-DR ${ }^{-}$subset. This suppression is contact-dependent and these cells do not produce IL-10. The action of these cells is unique from that of HLA-DR ${ }^{-}$Tregs, which induce a later, less vigorous suppression of Tresp, and which rely on both cell-contact and IL-10 secretion to inhibit Tresp activation [59]. Suppression by HLA-DR ${ }^{-}$Tregs induce Tresp to secrete IL-10 and therefore cause these cells to become suppressive themselves [58]. Such infectious tolerance is unique to the HLA-DR ${ }^{-}$Treg subset, and may reflect contamination of this population with adaptive Tregs.

\section{Strength of signal and human Treg suppression}

Human Tregs must be activated through their TCR in order to be functionally suppressive $[60,61]$, although once activated, these cells do not need to be viable in co-culture in order to mediate Tresp suppression. The effective outcome of Tresp suppression is also dictated by the quality of T cell stimulation, as the strength of stimuli applied to the Tresp population has a strong influence on whether suppression or proliferation will occur during Treg:Tresp coculture. Tresp activated in the presence of strong costimulation are refractory to Treg suppression, as are Tresp supplemented with growth promoting cytokines [51,62]. Furthermore, increasing the strength of TCR signal increases the resistance of Tresp to regulation [62]. This data suggests that human Tregs can only suppress Tresp activated with low signal strength. In highly inflammatory environments, where Tresp are activated with high signal strength, human Tregs are unable to prevent Tresp proliferation and cytokine secretion.

\section{Treg frequency in patients with MS}

Autoreactive $\mathrm{T}$ cells isolated from patients with autoimmune disease have a lower threshold of activation than Tresp in normal subjects [63]. As discussed previously in this chapter, both the activation status of the Tresp and Treg can impact suppression ex vivo; we therefore sought to characterize Tregs in patients with MS as compared to healthy controls. Our group hypothesized that a lower threshold of Treg activation could reduce effector function or cause deficiency in Treg generation in MS patients. We first compared frequency of $\mathrm{CD} 4{ }^{+} \mathrm{CD} 25^{\mathrm{hi}}$ regulatory $\mathrm{T}$ cells derived from a group of untreated patients who have relapsing/remitting MS with those from age-matched healthy control subjects [64]. We were unable to detect any differences in Treg frequency between patients and healthy controls in either the $\mathrm{CD} 4^{+} \mathrm{CD} 25^{\text {high }}$ or the bulk CD $25^{+}$populations. Vandenbark and coworkers, however, showed decreases in FoxP3 levels in Tregs isolated from patients with MS, and found that this decrease correlated with Treg loss of function [65]. Additionally, Venken et al, reported that relapsingremitting (but not secondary progressive) MS patients express lower levels of FoxP3 than healthy controls [66]. It is not yet clear from these studies whether lower FoxP3 expression in MS patients is due to a reduced frequency of FoxP3 $3^{+}$cells in the $\mathrm{CD} 4{ }^{+} \mathrm{CD} 25^{\text {hi }} \mathrm{T}$ cell population or due to decreased FoxP3 expression at a cellular level. 


\section{Impaired Treg function in patients with MS}

We isolated highly purified $\mathrm{CD} 4^{+} \mathrm{CD} 25^{\text {high }}$ Tregs and $\mathrm{CD} 4^{+} \mathrm{CD} 25^{\text {neg }}$ Tresp from untreated patients with relapsing/remitting MS and age-matched healthy control subjects to assay Treg function, as well as frequency. Responder cells from both MS patients and healthy individuals responded similarly in a dose-dependent fashion to varying concentrations of plate-bound $\alpha \mathrm{CD} 3$ monoclonal antibody $(\mathrm{mAb})$. As expected, Tregs isolated from both groups were anergic to stimulation at all doses of plate-bound $\alpha \mathrm{CD} 3$. This anergy indicates that $\mathrm{CD} 4{ }^{+} \mathrm{CD} 25^{\mathrm{hi}} \mathrm{T}$ cells isolated from patients with MS are not activated CD25 ${ }^{+} \mathrm{T}$ cells; such cells would not exhibit regulatory activity but rather enhance proliferation.

To quantify regulatory function, we co-cultured Tregs with autologous Tresp at multiple ratios (responder:suppressor ratios of 1:1,1:1/2,1:1/4, and 1:1/8). Consistent with previous reports, Tregs isolated from our healthy controls consistently suppressed proliferation at a 1:1 ratio and increasing the ratio of responder:suppressor T cells reduced suppression. In striking contrast, we found that Tregs isolated from the circulation of patients with MS, although normal in frequency, poorly inhibited Tresp proliferation. In these cultures, the secretion of the Th1 cytokine IFN- $\gamma$ by Tresp was suppressed by healthy Tregs but not by Tregs derived from patients with MS. The suppressive cytokine IL-10 was secreted in these cultures, predominantly by the $\mathrm{CD} 4^{+} \mathrm{CD} 25^{-}$Tresp, but we could not determine a role for IL-10 in mediating suppression by $\mathrm{CD} 4{ }^{+} \mathrm{CD} 25^{\text {high }}$ Tregs. Additionally, blocking IL-10 or TGF- $\beta$ did not result in loss of suppressor function in these cells.

The loss of Treg functionality in MS patients could be due to a decrease in $\mathrm{CD} 4{ }^{+} \mathrm{CD} 25^{\mathrm{hi}}$ Treg performance or an increase in inhibition resistance by activated Tresp. Thus, we performed mixing experiments in which patient and control Treg were co-cultured with the autologous and the converse Tresp isolated from either healthy subjects or patients with MS. Regulatory T cells from patients with MS could not suppress the proliferative response of target responder $\mathrm{T}$ cells from either patients or healthy controls (suppression 23\%). In the reciprocal experiments, Treg from healthy controls suppressed the proliferative response of Treg isolated from both controls and patients with MS (suppression 78\%). These data indicate that patients with MS do suffer a regulatory defect, which is due to impaired Treg function, but we have not yet determined the mechanism of this functional defect.

Our group has also characterized functional abnormalities in other regulatory $\mathrm{T}$ cell populations in patients with MS, specifically CD46-mediated Tr1 regulatory T cells [67]. CD46 is a newly defined costimulatory molecule that can induce a Tr1 phenotype and secretion of the antiinflammatory cytokine IL-10. We observed striking defects in the induction of Tr1 cells with CD46 co-stimulation, in their ability to secrete IL-10 (but not INF- $\gamma$ ) in patients with MS compared with healthy subjects [67].

\section{CD62L expression on $\mathrm{CD} 4{ }^{+} \mathrm{CD} 25^{\text {hi }}$ regulatory $\mathrm{T}$ cells}

Although there were no differences in the frequency of $\mathrm{CD} 4{ }^{+} \mathrm{CD} 25^{\text {hi }} \mathrm{T}$ cells or in their proliferative or cytokine secretion in response to different stimuli between healthy subjects and patients with MS, it was important to determine whether an increase in the frequency of activated $\mathrm{CD} 4^{+} \mathrm{T}$ cells in the circulation was diluting the regulatory $\mathrm{CD} 4^{+} \mathrm{CD} 25^{\text {hi }} \mathrm{T}$ cells. Therefore, we used CD62L expression to further purify regulatory from the activated $\mathrm{T}$ cells, because L-selectin expression is downregulated upon activation. We isolated $\mathrm{CD} 4{ }^{+} \mathrm{CD} 25^{\mathrm{hi}}$ $\mathrm{CD}_{2} \mathrm{~L}^{+}$and total $\mathrm{CD} 4{ }^{+} \mathrm{CD} 25^{\mathrm{hi}} \mathrm{CD} 62 \mathrm{~L}^{+}$regulatory $\mathrm{T}$ cells from healthy subjects and patients with MS. Whereas in the healthy controls both populations were able to suppress the proliferative response to anti-CD3 stimulation, the Tregs isolated from patients with MS, although further depleted of the potentially activated $\mathrm{CD}_{2} \mathrm{~L}^{-} \mathrm{T}$ cells, were still unable to 
inhibit the proliferation of the Tresp population. These data strongly confirm a defect in the highly purified regulatory subset in patients with MS.

\section{Tregs and autoimmune disease}

The inability of human Tregs to suppress strongly activated Tresp in inflammatory environments has major implications in autoimmune disease. Autoreactive T cells isolated from patients with autoimmune disease have a lower threshold of activation when compared to cells isolated from healthy individuals. Patient-derived Tresp, however, have not been shown to be resistant to suppression; instead, our group and others have demonstrated defects in the function of peripheral blood $\mathrm{CD} 4{ }^{+} \mathrm{CD} 25^{\text {high }}$ Tregs in patients with multiple sclerosis (MS) (Figure 2), type I diabetes (T1D), psoriasis, autoimmune myasthenia gravis and rheumatoid arthritis (RA) [64,68-71].

The loss of function in autoimmune Tregs has been correlated with decreases in FoxP3 levels. Indeed, the immunodeficiency syndrome IPEX (immune dysregulation, polyendocrinopathy, enteropathy, X-linked syndrome), which is associated with autoimmune disease in multiple endocrine organs, is caused by mutations in the foxp3 gene [72]. Impairment of FoxP3 protein expression results in massive inflammatory pathology, in both humans and mouse. Most human autoimmune diseases, however, are complex genetic disorders comprised of multiple common allelic variants that can, in combination with environmental factors, lead to development of a pathologic response. Genome wide association scans have recently been published for patients with MS, T1D, and RA $[73,74]$. While these scans have failed to implicate a significant genetic role for foxpP3 in autoimmune disease, the major growth factor receptor for Tregs, the IL-2R $\alpha$ chain, has been clearly linked to MS and type I diabetes. Thus, these and the other allelic variants such as in the IL-7R $\alpha$ chain and CD58 may partially regulate Treg function in patients with autoimmune disease.

\section{Regulatory T cells and MS therapy}

Unlike in other autoimmune diseases, such as diabetes where the majority of the pancreatic islet cells are destroyed with clinical presentation, patients with MS are now identified relatively early in the disease, allowing manipulation of the immune system in disease prevention. Earlier in this chapter, we discussed in detail how the cells of the immune system are involved in damaging the CNS, resulting in the varied and debilitating symptoms of MS. An ideal therapy would seek to prevent the initiation of such attacks, to halt such attacks if in progress, and optimally, to reverse the damage done. Because the disease is the result of a dysregulated immune system, rational therapy would be aimed at correcting the underlying dysfunction. Immunotherapy refers to therapy seeking to alter the immune response to prevent or treat the disease in question. Thus, unlike treatment of other diseases, such as some types of cancer, in the case of MS almost any promising therapy will be termed an immunotherapy, due to the autoimmune nature of the disease. The potential for Treg cells to control CNS autoimmunity has been well documented in experimental models. Treg cells administered to mice can significantly reduce EAE severity [75], and have been shown to accumulate within the CNS during the recovery [76]. In relapsing-remitting EAE models, not only does depletion of Treg cells increase acute-phase severity, but it also prevents remission [77,78]. The key role that Treg cells play in the EAE model identifies this cell type as a major potential target for human immunotherapy.

The origin of the observed defect of suppressive function of Tregs in relapsing-remitting MS patients has not yet been elucidated. We have recently reported using large-scale genomic screens, that certain alleles of the genes encoding for IL $2 \alpha$ and IL7R $\alpha$ chains are associated with increased risk for MS [73]. This supports the idea that polymorphisms within genes related to the regulation of the immune response, and to Treg cells in particular (which express high 
levels of CD25) are important factors in MS. While this data suggests that the functional alterations of Treg cells in these patient groups could be linked to genetic factors, as with any in vitro observation, clinical trials targeting the function or frequency of Tregs and any association with clinical responses, be it autoimmune disease, HIV, or with tissue transplantation must be performed. Moreover, the lack of a role for cytokine in these in vitro studies of these $\mathrm{CD} 4{ }^{+} \mathrm{CD} 25^{\text {hi }}$ Treg cells should not exclude a critical role for IL-10, TGF- $\beta$, or other cytokines in the combined in vivo mechanisms of these regulatory cells. The limitations of in vitro analyses enhance the difficulty of understanding human disease, as the assays are likely to reflect only single in vivo events rather than the culmination of all biologic mechanisms that would operate in vivo; that is, these models only observe what is asked of them.

Nevertheless, the apparently parallel observations of $\mathrm{CD} 4{ }^{+} \mathrm{CD} 25^{+}$Tregs in animal models and humans indicate that suppressor cells act as central regulators of immune responses in human diseases.

We have shown that the regulatory function of the $\mathrm{CD} 4^{+} \mathrm{CD} 25^{\text {hi }} \mathrm{T}$-cell subpopulation is defective in patients with MS [64]. These cells are thought to have a role in immune homeostasis, that is, in tempering the immune response and preventing postimmune response inflammation, etc. Therefore, a functional lack of $\mathrm{CD} 4{ }^{+} \mathrm{CD} 25^{\text {hi }} \mathrm{T}$ cells in MS patients is likely to contribute to the pathophysiology of the disease. Thus, it follows from this, and from experimental models of MS, that reversing this lack of functional regulatory cells would be a viable and productive treatment avenue. However, effective long-term treatment is likely to be more complex. It has been recently shown, at least in mouse models, that myelin-specific functional Treg cells accumulate in the CNS but fail to control autoimmune inflammation [79]. The authors speculate that in order for Tregs to effectively control autoimmune reactions in the CNS, it may be necessary to control tissue inflammation. Unfortunately, while a great deal is known about the requirements and characteristics of these cells in mice, much less is known about them in humans. Once we have a more complete understanding of the regulatory T-cell subsets in humans, this area will be an attractive one for intervention or immunotherapy with a goal of increasing the number and/or the functionality of these T cells in MS patients.

\section{Acknowledgments}

Work was supported by the NIH grants: UO1DK6192601, RO1NS2424710, PO1AI39671, and PO1NS38037; and grants from the National Multiple Sclerosis Society: RG2172C9 and RG3308A10, and from the 2004 FOCIS Centers of Excellence Amgen Award.

\section{References}

1. Mackay RP, Myrianth NC. Multiple Sclerosis in Twins and Their Relatives - Final Report. Arch Neurol 1966;15(5):449-457. [PubMed: 5955946]

2. Risch N. Linkage Strategies for Genetically Complex Traits. I. Multilocus Models. Am J Hum Gene 1990;46:222-228.

3. McCarthy MI. Susceptibility gene discovery for common metabolic and endocrine traits. J Mol Endocrin 2002;28:1-17.

4. Kabat EA, Glusman M, Knaub V. Quantitative Estimation of the Albumin and Gamma-Globulin in Normal and Pathologic Cerebrospinal Fluid by Immunochemical Methods. American Journal of Medicine 1948;4(5):653-662. [PubMed: 18856761]

5. Rivers TM, Sprunt DH, Berry GP. Observations on attempts to produce acute disseminated encephalomyelitis in monkeys. J Exp Med 1933;58(1):39-U58.

6. Goverman J, et al. Transgenic Mice That Express a Myelin Basic Protein-Specific T-Cell Receptor Develop Spontaneous Autoimmunity. Cell 1993;72(4):551-560. [PubMed: 7679952]

7. Miller DH, et al. A controlled trial of natalizumab for relapsing multiple sclerosis. New Eng J Med 2003;348:15-23. [PubMed: 12510038] 
8. Ota K, et al. T-Cell Recognition of an Immunodominant Myelin Basic-Protein Epitope in MultipleSclerosis. Nature 1990;346(6280):183-187. [PubMed: 1694970]

9. Pette M, et al. Myelin Basic Protein-Specific Lymphocyte-T Lines from Ms Patients and HealthyIndividuals. Neurology 1990;40(11):1770-1776. [PubMed: 1700336]

10. Martin R, et al. Fine Specificity and Hla Restriction of Myelin Basic Protein-Specific Cytotoxic TCell Lines from Multiple-Sclerosis Patients and Healthy-Individuals. J Immunol 1990;145(2):540_ 548. [PubMed: 1694881]

11. Hahn M, Nicholson MJ, Pyrdol J, Wucherpfennig KW. Unconventional topology of self peptidemajor histocompatibility complex binding by a human autoimmune T cell receptor. Nat Immunol 2005;6(5):490-496. [PubMed: 15821740]

12. Ausubel LJ, et al. Complementary mutations in an antigenic peptide allow for crossreactivity of autoreactive T-cell clones. PNAS USA 1996;93(26):15317-15322. [PubMed: 8986809]

13. Zhang X, et al. Degenerate TCR recognition and dual DR2 restriction of autoreactive T cells: Implications for the initiation of the autoimmune response in multiple sclerosis. Euro J Immunol 2008;38(5):1297-1309.

14. Fujinami RS, Oldstone MB. Amino acid homology between the encephalitogenic site of myelin basic protein and virus: mechanism for autoimmunity. Science 1985;230(4729):1043-1045. [PubMed: 2414848]

15. Wucherpfennig KW, Strominger JL. Molecular Mimicry in T-Cell-Mediated Autoimmunity - Viral Peptides Activate Human T-Cell Clones Specific for Myelin Basic-Protein. Cell 1995;80(5):695705. [PubMed: 7534214]

16. Hemmer B, et al. Identification of high potency microbial and self ligands for a human autoreactive class II-restricted T cell clone. J Exp Med 1997;185(9):1651-1659. [PubMed: 9151902]

17. Lang HL, et al. A functional and structural basis for TCR cross-reactivity in multiple sclerosis. Nat Immunol 2002;3(10):940-943. [PubMed: 12244309]

18. Lenz DC, et al. A Chlamydia pneumoniae-specific peptide induces experimental autoimmune encephalomyelitis in rats. J Immunol 2001;167(3):1803-1808. [PubMed: 11466406]

19. Fujinami RS, et al. Molecular mimicry in virus infection: crossreaction of measles virus phosphoprotein or of herpes simplex virus protein with human intermediate filaments. PNAS USA 1983;80(8):2346-2350. [PubMed: 6300911]

20. Croxford JL, Anger HA, Miller SD. Viral delifery of an epitope from Haemophilus influenzae induces central nervous system autoimmune disease by molecular mimicry. J Immunol 2005;174(2):907917. [PubMed: 15634913]

21. Ascherio A, Munger KL. Environmental risk factors for multiple sclerosis. Part I: the role of infection. Ann Neuro 2007;61(4):288-299.

22. de Jager PL, et al. Integrating risk factors: HLA-DRB1*1501 and Epstein-Barr virus in multiple sclerosis. Neurology 2008;70(13 part 2):1113-1118. [PubMed: 18272866]

23. Lehmann PV, et al. Spreading of T Cell Autoimmunity to Cryptic Determinants of an Autoantigen. Nature 1992;358(6382):155-157. [PubMed: 1377368]

24. Ellmerich $\mathrm{S}$, et al. Disease-related epitope spread in a humanized T cell receptor transgenic model of multiple sclerosis. Eur J Immunol 2004;34(7):1839-1848. [PubMed: 15214032]

25. Trapp BD, et al. Axonal transection in the lesions of multiple sclerosis. New Eng J Med 1998;338 (5):278-285. [PubMed: 9445407]

26. Lucchinetti CF, et al. Distinct patterns of multiple sclerosis pathology indicates heterogeneity in pathogenesis. Brain Pathology 1996;6(3):259-274. [PubMed: 8864283]

27. Wucherpfennig KW, et al. T-Cell Receptor V-Alpha-V-Beta Repertoire and Cytokine GeneExpression in Active Multiple-Sclerosis Lesions. J Exp Med 1992;175(4):993-1002. [PubMed: 1348083]

28. Traugott U, Reinherz EL, Raine CS. Multiple-Sclerosis - Distribution of T-Cell Subsets within Active Chronic Lesions. Science 1983;219(4582):308-310. [PubMed: 6217550]

29. Hauser SL, et al. Immunohistochemical Analysis of the Cellular Infiltrate in Multiple-Sclerosis Lesions. Annals of Neurology 1986;19(6):578-587. [PubMed: 3524414] 
30. Wucherpfennig KW, et al. Gamma-Delta T-Cell Receptor Repertoire in Acute Multiple-Sclerosis Lesions. PNAS USA 1992;89(10):4588-4592. [PubMed: 1374907]

31. Prineas JW, Wright RG. Macrophages, Lymphocytes, and Plasma-Cells in Perivascular Compartment in Chronic Multiple-Sclerosis. Laboratory Investigation 1978;38(4):409-421. [PubMed: 205724]

32. Prineas J. Pathology of Early Lesion in Multiple-Sclerosis. Hum Path 1975;6(5):531-554. [PubMed: 170186]

33. Becher B, et al. Soluble tumor necrosis factor receptor inhibits interleukin 12 production by stimulated human adult microglial cells in vitro. J Clin Invest 1996;98(7):1539-1543. [PubMed: 8833901]

34. Anderson AC, Anderson DE, Bregoli L, Hastings WD, Kassam N, Lei C, Chandwaskar R, Karman J, Su EW, Hirashima M, Bruce JN, Kane LP, Kuchroo VK, Hafler DA. TIM-3 is expressed by cells of the innate immune system and promotes tissue inflammation. Science 2007;318(5853):11411143. [PubMed: 18006747]

35. Lehnardt S, et al. Activation of innate immunity in the CNS triggers neurodegeneration through a Toll-like receptor 4-dependent pathway. PNAS USA 2003;100(14):8514-8519. [PubMed: 12824464]

36. Monje ML, Toda H, Palmer TD. Inflammatory blockade restores adult hippocampal neurogenesis. Science 2003;302(5651):1760-1765. [PubMed: 14615545]

37. Ekdahl CT, et al. Inflammation is detrimental for neurogenesis in adult brain. PNAS USA 2003;100 (23):13632-13637. [PubMed: 14581618]

38. Baranzini SE, et al. Transcriptional analysis of multiple sclerosis brain lesions reveals a complex pattern of cytokine expression. J Immunol 2000;165(11):6576-6582. [PubMed: 11086101]

39. Mycko MP, et al. Microarray gene expression profiling of chronic active and inactive lesions in multiple sclerosis. Clinical Neurology and Neurosurgery 2004;106(3):223-229. [PubMed: 15177772]

40. Aloisi F, et al. IL-12 production by central nervous system microglia is inhibited by astrocytes. $\mathrm{J}$ Immunol 1997;159(4):1604-1612. [PubMed: 9257819]

41. Ledeboer A, et al. Interleukin-10, interleukin-4, and transforming growth factor-beta differentially regulate lipopolysaccharide-induced production of pro-inflammatory cytokines and nitric oxide in co-cultures of rat astroglial and microglial cells. Glia 2000;30(2):134-142. [PubMed: 10719355]

42. Onuki I, et al. Axonal degeneration is an early pathological feature in autoimmune-mediated demyelination in mice. Microscopy Research and Technique 2001;52(6):731-739. [PubMed: 11276125]

43. Ayers MM, et al. Early glial responses in murine models of multiple sclerosis. Neurochem Int 2004;45 (23):409-419. [PubMed: 15145555]

44. Trajkovic V, et al. Astrocyte-induced regulatory T cells mitigate CNS autoimmunity. Glia 2004;47 (2):168-179. [PubMed: 15185395]

45. Roncarolo MG, et al. Type 1 T regulatory cells. Imm Rev 2001;182:68-79.

46. Pullen AM, Marrack P, Kappler JW. Evidence That Mls-2 Antigens Which Delete V-Beta-3+T-Cells Are Controlled by Multiple Genes. J Immunol 1989;142(9):3033-3037. [PubMed: 2496158]

47. Sakaguchi S. Regulatory T cells: Key controllers of immunologic self-tolerance. Cell 2000;101(5): 455-458. [PubMed: 10850488]

48. Shevach EM, et al. Control of T-cell activation by CD4(+) CD25(+) suppressor T cells. Imm Rev 2001;182:58-67.

49. Sakaguchi S, et al. Organ-Specific Autoimmune-Diseases Induced in Mice by Elimination of T-Cell Subset .1. Evidence for the Active Participation of T-Cells in Natural Self-Tolerance - Deficit of a T-Cell Subset as a Possible Cause of Autoimmune-Disease. J Exp Med 1985;161(1):72-87. [PubMed: 3871469]

50. Read S, Malmstrom V, Powrie F. Cytotoxic T lymphocyte-associated antigen 4 plays an essential role in the function of $\mathrm{CD} 25(+) \mathrm{CD} 4(+)$ regulatory cells that control intestinal inflammation. J Exp Med 2000;192(2):295-302. [PubMed: 10899916]

51. Baecher-Allan C, et al. CD4+CD25(high) regulatory cells in human peripheral blood. J1 Immunol 2001;167(3):1245-1253. 
52. Dieckmann D, et al. Ex vivo isolation and characterization of CD4(+)CD25(+) T cells with regulatory properties from human blood. J Exp Med 2001;193(11):1303-1310. [PubMed: 11390437]

53. Wang J, et al. Transient expression of FOXP3 in human activated nonregulatory CD4+ T cells. Eur J of Immunol 2007;37:129-138. [PubMed: 17154262]

54. Fontenot JD, et al. Regulatory $\mathrm{T}$ cell lineage specification by the forkhead transcription factor foxp3. Immunity 2005;22:329-341. [PubMed: 15780990]

55. Liu W, et al. CD127 expression inversely correlates with FoxP3 and suppressive function of human CD4+ Treg cells. J Exp Med 2006;203:1701-1711. [PubMed: 16818678]

56. Seddiki N, et al. Expression of interleukin (IL)-2 and IL-7 receptors discriminates between human regulatory and activated T cells. J Exp Med 2006;203:1693-1700. [PubMed: 16818676]

57. Ruprecht CR, et al. Coexpression of CD25 and CD27 identifies FoxP3+ regulatory T cells in invlamed synovia. J Exp Med 2005;201:1793-1803. [PubMed: 15939793]

58. Baecher-Allan CM, Wolf E, Hafler DA. MHC class II expression identifies functionally distinct human regulatory T cells. J Immunol 2006;176:4622-4631. [PubMed: 16585553]

59. Baecher-Allan CM, Hafler DA. Human regulatory T cells and their role in autoimmune disease. Imm Rev 2006;212:203-216.

60. Dieckmann D, et al. Human CD4(+)CD25(+) regulatory, contact-dependent T cells induce interleukin 10-producing contact-independent type 1-like regulatory T cells. J Exp Med 2002;196:247-253. [PubMed: 12119349]

61. Jonuleit $\mathrm{H}$, et al. Infectious tolerance: human CD25(+) regulatory T cells convey suppressor activity to conventional CD4(+) helper cells. J Exp Med 2002;196:255-260. [PubMed: 12119350]

62. Baecher-Allan C, Viglietta V, Hafler DA. Inhibition of human CD4(+)CD25(+high) regulatory T cell function. J Immunol 2002;169(11):6210-6217. [PubMed: 12444126]

63. Reijonen H, et al. Detection of GAD65-specific T-cells by major histocompatibility complex class II tetramers in type 1 diabetic patients and at-risk subjects. Diabetes 2002;51(5):1375-1382. [PubMed: 11978633]

64. Viglietta $\mathrm{V}$, et al. Loss of functional suppression by CD4+CD25+ regulatory $\mathrm{T}$ cells in patients with multiple sclerosis. J Exp Med 2004;199(7):971-9. [PubMed: 15067033]

65. Huan J, et al. Decreased FOXP3 levels in multiple sclerosis patients. J Neurosci Res 2005;81(1):4552. [PubMed: 15952173]

66. Venken K, et al. Secondary progressive in contrast to relapsing-remitting multiple sclerosis patients show a normal CD4+CD25+ regulatory T-cell function and FOXP3 expression. J Neurosci Res 2006;83(8):1432-46. [PubMed: 16583400]

67. Astier AL, et al. Alterations in CD46-mediated Tr1 regulatory T cells in patients with multiple sclerosis. J Clin Invest 2006;116(12):3252-7. [PubMed: 17099776]

68. Cao D, et al. Isolation and functional characterization of regulatory CD25brightCD4+ T cells from the target organ of patients with rheumatoid arthritis. J Immunol 1998;160:1532-1538. [PubMed: 9570577]

69. Sugiyama H, et al. Dysfunctional blood and target tissue CD4+CD25high regulatory T cells in psoriasis: mechanism underlying unrestrained pathogenic effector T cell proliferation. J Immunol 2005;174:164-173. [PubMed: 15611238]

70. Lindley S, et al. Defective suppressor function in CD4(+)CD25(+) T-cells from patients with type 1 diabetes. Diabetes 2005;54:92-99. [PubMed: 15616015]

71. Balandina A, et al. Analysis of CD4+CD25+ cell population in the thymus from myasthenia gravis patients. Ann NY Acad Sci 2003;998:275-277. [PubMed: 14592885]

72. Gambineri E, et al. Immune dysregulation, polyendocrinopathy, enteropathy, and X-linked inheritance (IPEX), a syndrome of systemic autoimmunity caused by mutations of FOXP3, a critical regulator of T-cell homeostasis. Curr Opin Rheum 2003;15:430-435.

73. Hafler DA, et al. Risk alleles for multiple sclerosis identified by a genomewide study. New Eng J Med 2007;357:851-862. [PubMed: 17660530]

74. The Wellcome Trust Cast Consortium. Genome-wide association study of 14,000 cases of seven common diseases and 3,000 shared controls. Nature 2007;447:661-678. [PubMed: 17554300] 
75. Kohm AP, et al. Cutting edge: CD4+CD25+ regulatory T cells suppress antigen-specific autoreactive immune responses and central nervous system inflammation during active experimental autoimmune encephalomyelitis. J Immunol 2002;169(9):4712-6. [PubMed: 12391178]

76. McGeachy MJ, Stephens LA, Anderton SM. Natural recovery and protection from autoimmune encephalomyelitis: contribution of CD4+CD25+ regulatory cells within the central nervous system. J Immunol 2005;175(5):3025-32. [PubMed: 16116190]

77. Gartner D, et al. CD25 regulatory T cells determine secondary but not primary remission in EAE: impact on long-term disease progression. J Neuroimmunol 2006;172(12):73-84. [PubMed: 16360886]

78. Zhang X, et al. Recovery from experimental allergic encephalomyelitis is TGF-beta dependent and associated with increases in CD4+LAP+ and CD4+CD25+ T cells. Int Immunol 2006;18(4):495503. [PubMed: 16540527]

79. Korn T, et al. Myelin-specific regulatory T cells accumulate in the CNS but fail to control autoimmune inflammation. Nat Med 2007;13(4):423-31. [PubMed: 17384649] 